\title{
Verticillium dahliae-Eggplant as the Pathosystem Model to Reveal Biocontrol Potential of three Trichoderma spp in Greenhouse Conditions
}

\author{
Wafaa Mokhtari ${ }^{1 *}$, Mohamed Achouri ${ }^{1}$, Abdellah Remah $^{1}$, and Hassan Boubaker ${ }^{2}$ \\ ${ }^{1}$ Plant Protection Department, Institut Agronomique et Vétérinaire Hassan II, Agadir, Morocco; ${ }^{2}$ Biology \\ Department, School of Sciences, University Ibn Zohr, Agadir, Morocco
}

Received: January 17, 2018 / Accepted: February 17, 2018

\begin{abstract}
In this study three Trichoderma species isolated from Moroccan soil (natural and agricultural habitats) were investigated for their biocontrol potential against virulent Verticillium dahliae on eggplant in green house conditions based on dipping root approach. Evaluation of biocontrol efficacy of Trichoderma spp. demonstrated effective potential of Trichoderma on reducing Verticilium disease on eggplant cultivars. Disease assessment was established by measuring disease incidence in root units (DI-RU) and in the above ground of eggplant cultivars (DI- AU). DI-RU\% was recorded at $12.5 \%, 25.0 \%, 31.3 \%$ and $37.5 \%$ in T3, TC, T1 and $\mathrm{T} 2$ treatment respectively where DI-RU recorded in Tm2 controls; eggplants inoculated with Verticillium only was equal to $100.0 \%$. Whereas, disease incidence outcomes in aerial part was DI- $\mathrm{AU}=\mathbf{1 0 0 . 0 \%}$ in treatments $\mathrm{TC}$ and $\mathrm{T} 2,87.5 \%$ in $\mathrm{T} 1$ and $96.0 \%$ in $\mathrm{T} 3$ treatment where $\mathrm{Tm} 2$ controls were assessed with $100.0 \%$.
\end{abstract}

Keywords: Trichoderma, Biocontrol, Verticillium dahliae-eggplant pathosystem.

Abbreviations: g; gram, $\mathrm{cm}^{2}$; centimeter area, $\mathrm{cm}$; centimeter, DI; Disease Incidence, DI-AU; Disease incidence in Above ground units, DI-RU; Disease incidence in roots units; w/w; weight per weight, NPK; Nitrogen, Phosphor, Potassium, g/hl; gram per hectoliter, ${ }^{\circ} \mathrm{C}$; Celsius degree, $\mathrm{ml}$; milliliter.

"Corresponding author:w_mokhtari@yahoo.fr

\section{Introduction}

Verticillium dahliae is considered a highly polyphagous soil borne plant pathogen that attacks more than 400 vegetable species including Solanacea and Cucurbitaceae cultures and olive trees. Its polyphagous characteristic causes the attack of wide host range other than the original hosts what makes determination of host specificity and virulence of Verticillium isolates of paramount importance regarding its management (Tjamos, 1981). Verticillium is considered a chronic economic soil borne pathogen that causes vascular wilt and death in many plants in field and green house. No economic losses measure has been done till now in the cultural infestation with Verticilium in Morocco. However, it is estimated of billions of dollars annually losses all around the world (Pegg and Brady, 2002). Soil disinfection and genetic resistance are the major management strategies used to control Verticilium diseases. However, application of biocontrol agents like Trichoderma have become more and more used to control this soil borne pathogen due to negative effect of disinfectant on human health and environment (Cook and Baker, 1983).

Eggplant is a highly susceptible cultivar wilt and dies shortly after Verticillium dahliae invading and expressing first symptoms in it. In fact, Verticillium wilt has been reported to be the most destructive and prevalent diseases of eggplant. Therefore, eggplant-Verticillium is considered one of the best pathosystem models to evaluate resistance and/or tolerance to Verticilium wilt for designing the adequate and required disease management strategy (i.e. grafting, biocontrol application) in crop system. For instance, testing biocontrol potential of Trichoderma spp. using alike susceptible host controls would allow to iden- 
tify the effective biocontrol potential among Trichoderma isolates especially when selecting antagonistic candidates during biocontrol agent screening (Huisman and Gerik, 1989; Blestos et al., 2003).

Selecting antagonists for specific biocontrol of Verticillium diseases was based on screening potential antagonistic candidates like Trichoderma spp. Many studies demonstrated that Trichoderma spp. can inhibit growth or kill soil borne pathogens and revealed different antagonistic interactions between the antagonist Trichoderma and the pathogen in vitro and in vivo (Lumsden et al., 1993; Monte, 2001; Yang et al., 2010). Actually, few research work have focused on the biocontrol efficacy of Trichoderma spp against Verticillium in vivo though important antagonistic potential were involved against Verticillium dahliae when in vitro assays were applied.

In this study, we attempt to evaluate biocontrol potential of three Trichoderma species isolated from Moroccan soil (natural and agricultural habitats) in green house conditions based on root dipping technique.

\section{Material and Methods}

\section{Experimental Design for Biocontrol Treatments in Green- house Conditions}

Three species of Trichoderma identified at the species level (Mokhtari et al., 2017); Trichoderma afro-harzianum (T8A4), Trichoderma reseei (T9i12) and Trichoderma guizouhense (T4) were tested for their biocontrol efficacy against Verticillium dahliae-eggplant pathosystem. Eggplant cultivars were grown for three weeks on 77 peat trays. Seedlings were then transplanted into pots after their inoculation with fungi. Eggplant seedlings were transplanted in $3 \mathrm{~L}$ pots filled with sterile substrate at $3: 1 \mathrm{w} / \mathrm{w}$ peat to sand ratio. Substrate was fertilized using NPK and oligo-elements composition at $250 \mathrm{~g} / \mathrm{hl}$.

Experimental design was organized in four randomized complete blocs with four replicates in each experimental unit. That is, four pots were used in each experimental unit. Four treatments were tested; $\mathrm{T} 1$ was designated for treatment of cultivars inoculated with Trichoderma afro-harzianum (T814) and Verticillium dahliae, $\mathrm{T} 2$ was designated for treatment of cultivars inoculated with Trichoderma guizouhense (T4) and Verticillium dahliae and T3 was designated for treatment of cultivars inoculated with Trichoderma reesei (T9i12) and Verticillium dahliae. Controls were respectively; Tm1 was designated for healthy cultivars with non inoculated plants (negative controls), Tm2 was designated for cultivars inoculated with pathogen only (positive control). Verticillium has been known of its virulence and lethality towards woody and other crops. Pathogenicity test investigated in Tm2 controls allowed the exhibition of Verticillium dahliae virulence on susceptible eggplants (Schnathorst and Sibbett, 1971).

TC designated for treatment of cultivars inoculated with Trichoderma afro-harzianum extracted from a commercial product to be compared with other Trichoderma isolates.

\section{Obtaining Verticillium isolate for Pathogenic Test}

Branches and roots of olive trees diagnosed with wilt symptoms were collected, washed with tap water and disinfected one minute in $10 \%$ sodium hypochlorite.

Six small fragments from wilt branches and roots were washed in distilled di-ionized water for one more minute then inoculated in PDA and incubated at $19{ }^{\circ} \mathrm{C}$ in the dark for 10 days.

To obtain pure culture of Verticillium dahliae, mycelium of the fungus was collected from tissues baits with sterilized scalpel and inoculated into fresh PDA.

\section{Verticillium dahliae Microsclerotia Inoculum and Eggplant Root- Dipping Inoculation}

Microsclerotia were used exclusively as inoculum propagules to infest eggplant roots. Therefore, we used cellophane layer to produce uniform microsclerotia layer on potato dextrose agar (PDA, Difco) plate.

Cellophane plates with Verticillium microsclerotia were thereafter flooded with sterile di-ionized water and poured through $45 \mu \mathrm{m}$ stainless steel sieve to remove spores and hyphae. The contents on the sieve were transferred to sterile glass petri dishes and comminuted with a sterile razor. To prepare microsclerotia suspension, microsclerotia obtained were transferred in beaker filled with distilled di-ionized water. After deep vortex, number of microsclerotia was determined with serial dilution ranged from $10^{-1}$ to $10^{-6}$ to a final microsclerotia concentration of $10^{4}$ to $10^{5} / \mathrm{ml}$ (Atibalentja and Eastburn, 1997; Shiraishi et al., 2014; Dongfang et al., 2014). Eggplant roots cuts of 2 to $3 \mathrm{~cm}$ diameters long were inoculated in $10^{4}$ to $10^{5}$ $\mathrm{ml}$ of microsclerotia of Verticillium dahliae (Atibalentja and Eastburn, 1997; Gray et al., 1998).

\section{Disease Evaluation and Measurement of Leaves Surface Area (LSA)}

Disease assessment was estimated by measuring Disease Incidence (DI) of Verticillium dahliae as reported by Campbell and Neher (1994). Disease Incidence (DI) was measured as the percentage of number of plant units that are visibly diseased. Therefore, DI percentage was calculated as shown in the equation (1) (Campbell and Neher, 1994).

\section{Disease Incidence $=$ [Number of infected plant units] / [Total number of plants in the experiment] $x 100$ Equation 1}

Disease incidence was measured in above ground units corresponding to aerial parts of cultivars (DI-AU) and root units (DI-RU). DI- above ground Units was measured based on visible symptoms detected in the above-ground plant area. Therefore, symptoms were identified and measured in symptomatic vegetative tissues in leaves (i.e. typical bronze-yellowing, necrosis) and vascular tissues wilt. DI-RU was measured based on visible symptoms and signs in the diseased roots and crown (rots and/or discoloration) of related host plant. In fact, this 
method requires destructive sampling of plant, therefore, applied at the end of each experiment. Signs were basically examined based on the presence of pathogens' components like mycelium and propagules under microscopic observation (Campbell and Neher, 1994). In addition, plant parameters like Plant Height $(\mathrm{PH})$ in centimeter $(\mathrm{cm})$ and Leaf Surface Area (LSA) in square centimeters $\left(\mathrm{cm}^{2}\right)$ were also measured. These three parameters were assessed at the end of each experiment yet with non destructive method. Whereas, Root Dry Weight (RDW) and Plant Dry Weight (PDW) in gram (g) were measured at the end of each experiment with destructive sampling method (Benson and Baker, 1974; Campbell and Neher, 1994). In order to measure PDW, all plants were excavated from pots, roots were washed under running tap water to discard adhering substrate then exposed to the air at ambient temperature (25-30 ${ }^{\circ} \mathrm{C}$ ) until they dry. When PDW was measured, roots were cut at crown level and dried in drying chamber at $60{ }^{\circ} \mathrm{C}$ for three to four days to measure RDW. In order to measure leaves surface area (LSA) technique described by Breda (2003) was used. Since leaves are irregular, LSA was measured based on constant mass ratio calculation (Breda, 2003). At the end of each experiment leaves were collected, laid on the surface of A4 papers then their outlines were traced. Each leaf drawn on A4 paper was cut at the level of outline and A4 leaf drawing pieces were kept to be weighed. Surface area $\left(\mathrm{cm}^{2}\right)$ and the mass $(\mathrm{g})$ of a whole A4 paper were measured too. To calculate LSA $\left(\mathrm{cm}^{2}\right)$ of each A4 leaf drawing piece mass ratio was correlated to surface area $\left(\mathrm{cm}^{2}\right)$ of A4 paper as detailed in equation (2).

\section{[surface area of A4 paper $\left(\mathrm{cm}^{2}\right)$ ] [mass of A4 leaf drawing piece (g)]/[mass of A4 paper (g)] Euation 2}

\section{Results and Discussion}

\section{Reducing Verticillium Infestation on Eggplant Cultivars}

As mentioned in material and methods in vivo antagonistic assay was tested on eggplant. Similarly to previous antagonistic assay in green house, disease responses of Verticillium were assessed by measuring disease incidence in above ground units (DI- AU) and root units (DI-RU). For eggplant, disease assessment and antagonistic evaluation were performed three months after Trichoderma spp. treatments. On the whole, Trichoderma spp. treatments exhibit biocontrol efficacy reducing Verticillium disease in eggplant cultivars yet could not control effectively Verticillium severe symptoms on eggplant. As detailed in Figure 1 disease incidence in above ground Units $=100.0 \%$ in treatments TC and $\mathrm{T} 2,87.5 \%$ in $\mathrm{T} 1$ and $96.0 \%$ in $\mathrm{T} 3$ treatment.

It can be inferred from DI- above ground Unit results that Trichoderma spp. treatments each containing T1; T. afroharzianum, T2; T. guizouhense, T. reesei and TC; commercial Trichoderma were not able to suppress definitively the pathogen. In fact, different symptoms on the above-ground eggplant cultivars were detected; typical bronze-yellowing and necrosis of leaves veins and wilting as illustrated in Figures 2 and 3.

Moreover, significant decrease was recorded in leaves surfaces area (LSA) of cultivars in different treatments relatively

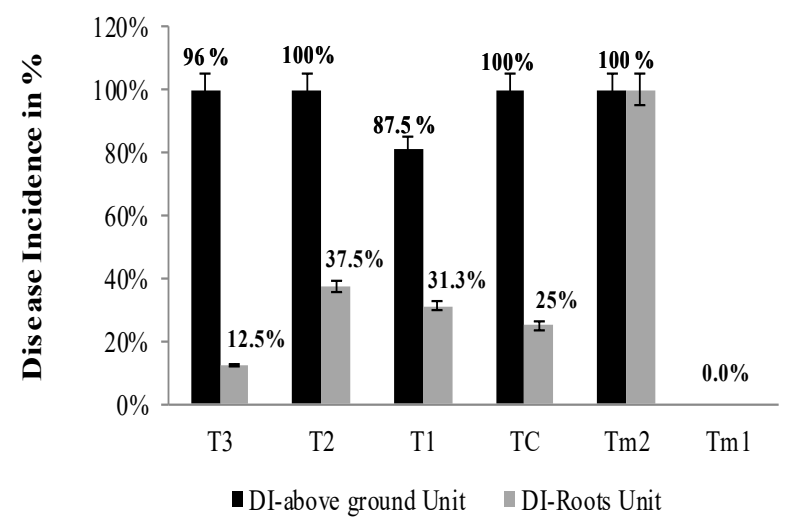

Figure 1. Disease incidence assessment in (\%) on Verticillium-eggplant pathosystem towards evaluation of effect of three antagonists; Trichoderma afroharzianum T1, Trichoderma reseei $\mathrm{T} 2$ and Trichoderma guizouhense T3. TC Trichoderma extracted from commercial product used as reference. Disease incidence was assessed in above ground units DI-AU and in roots; DI-RU.
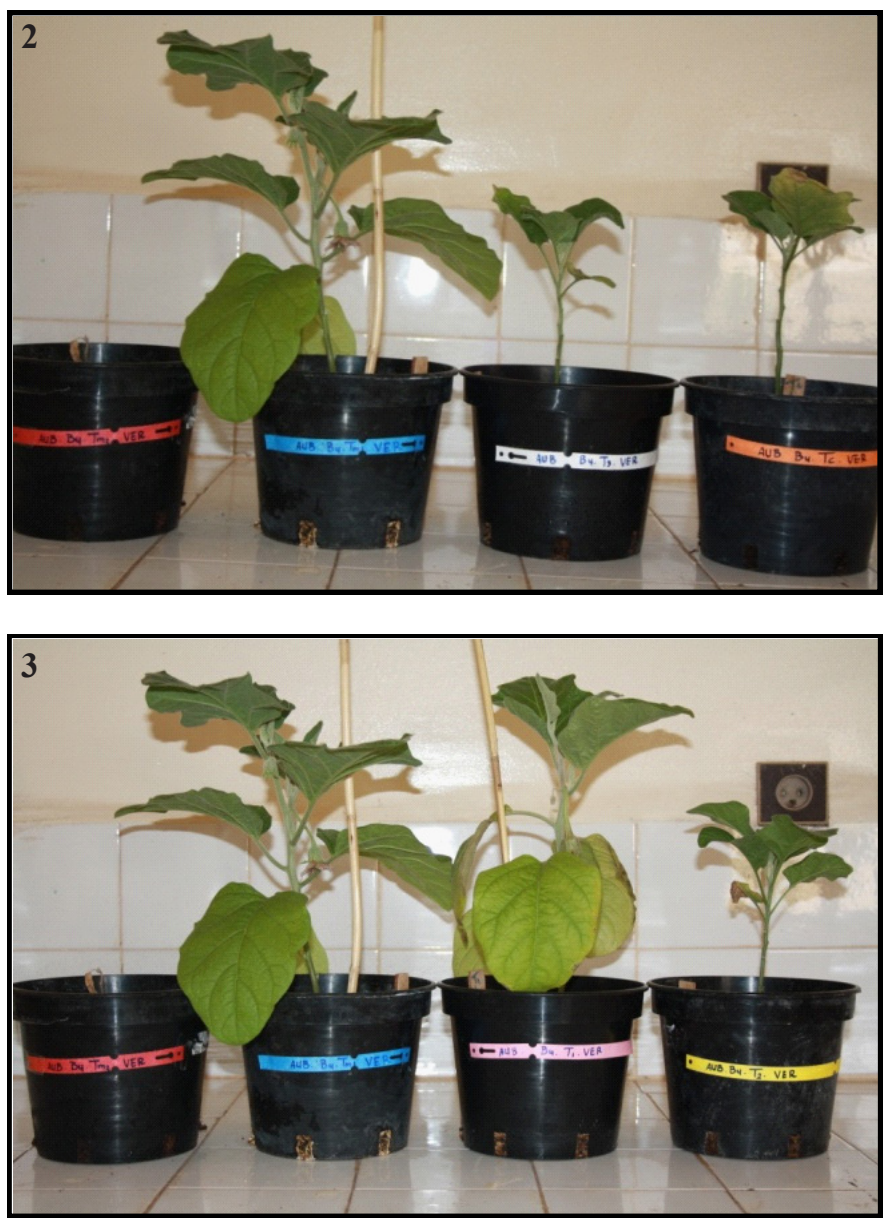

Figure 2 and 3. Trichoderma treatments of eggplant artificially infested with Verticillium microsclerotia; both figures represent above ground symptomatic eggplant treated with different Trichoderma spores suspenssions; T. afro-harzianum in $\mathrm{T} 1$ treatment, $T$. reseei in T2 and T. guizouhense in T3 and TC Trichoderma extracted from commercial product compared to healthy eggplants Tm1 and infested eggplant Tm2 controls. 

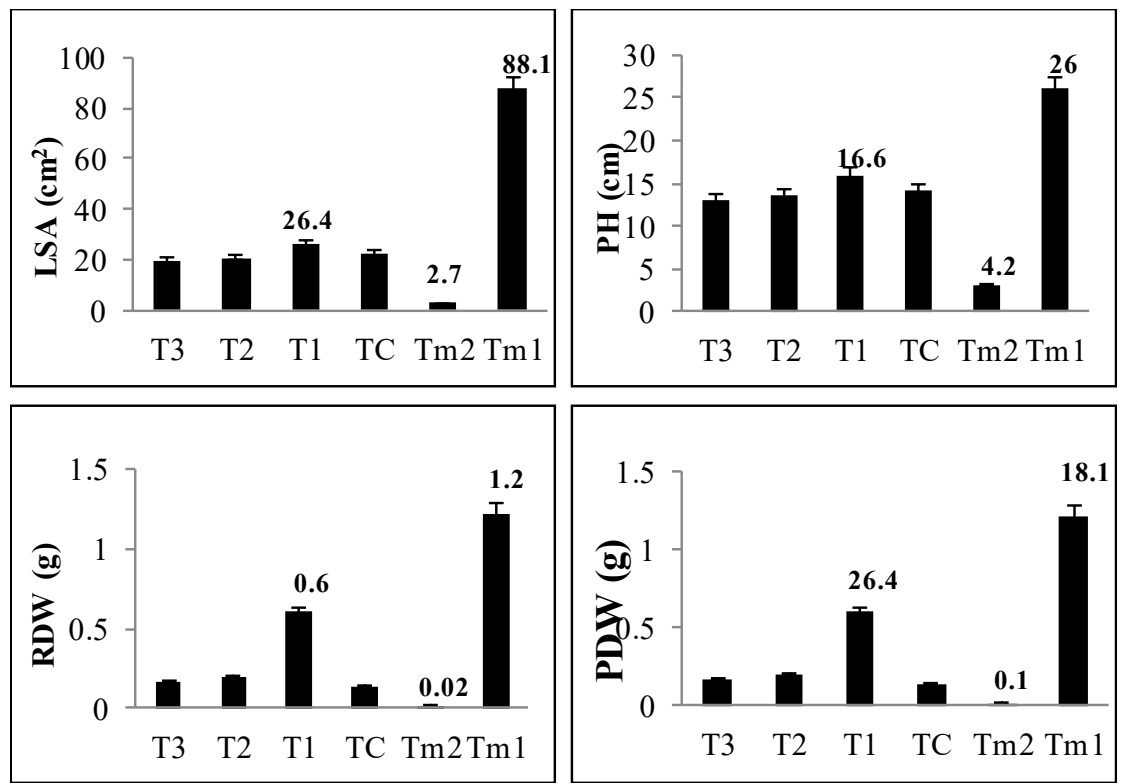

Figure 4. Evaluation of biocontrol efficacy of Trichoderma spp. on plant development parameters on Verticillium-eggplant pathosystem. Plant development parameters measured were; Leaves surface Area (LSA), Plant Height (PH), Root dry weight (RDW) and Plant dry weight (PDW).

compared to healthy plant Tm1. For instance LSA $=19.5 \mathrm{~cm}^{2}$ of eggplant occurred in T3 treatment while LSA $=88.1 \mathrm{~cm}^{2}$ occurred in healthy plant $\mathrm{Tm} 1,(P=0.000)$. LSA dramatically decreased to $2.7 \mathrm{~cm}^{2}$ in Tm2 controls (Figure 4).

Interestingly, there seems to be different asset between disease incidence assessed above-ground (DI-AU) and in root unit (DI-RU). DI-RU was recorded at $12.5 \%, 25.0 \%, 31.3 \%$ and $37.5 \%$ in T3, TC, T1 and T2 treatment respectively (Figure 1). Root Dry Weight (RDW) recorded in eggplant with different treatments was respectively 0.1 gram $(\mathrm{g})$ in TC, $0.2 \mathrm{~g}$ in T2 and $\mathrm{T} 3$ and $0.6 \mathrm{~g}$ in T1 compared to $1.2 \mathrm{~g} \mathrm{RDW}$ for healthy eggplant controls $\mathrm{Tm} 1$. It seems that roots weight was significantly affected by disease responses when infested with Verticillium with only $\mathrm{RDW}=0.2 \mathrm{~g}$ in $\mathrm{Tm} 2$ controls (for more details see Figures 4 and 5). In fact, Root dry weight recorded support the overall results obtained in DI-RU. It may be reasonable to suppose from DI-RU values that Trichoderma treatments were able to alleviate Verticillium root disease in eggplant. Moreover, from plant height $(\mathrm{PH})$ results, it can be deduced that eggplant height decreased at some extent. Yet, PH was maintained at somewhat remarkable level in treated eggplants compared to $\mathrm{Tm} 2$ controls. That is, $\mathrm{Tm} 2$ infested controls hardly reached their 4.2 centimeters $(\mathrm{cm})$ height whereas eggplant cultivars in T1 treatment containing T. afro-harzianum maintained their height at $16.6 \mathrm{~cm}(P=0.000)$. $\mathrm{PH}$ in eggplants in $\mathrm{T} 2, \mathrm{~T} 3$ and TC treatment noticeably reached $12.6,13$ to 13.5 centimeters respectively compared to $\mathrm{Tm} 1$ with $\mathrm{PH}=26 \mathrm{~cm},(P=0.000)$.

These results were in line with some of previous work on Verticillium biocontrol efficacy test in many crop-systems in green house and field conditions. For instance, Zheng et al (2011) tested biocontrol potential and efficacy of 105 antagonists including Trichoderma spp. in the $V$. dahliae-cotton pathosystem in green house conditions. In their work, Zheng et al

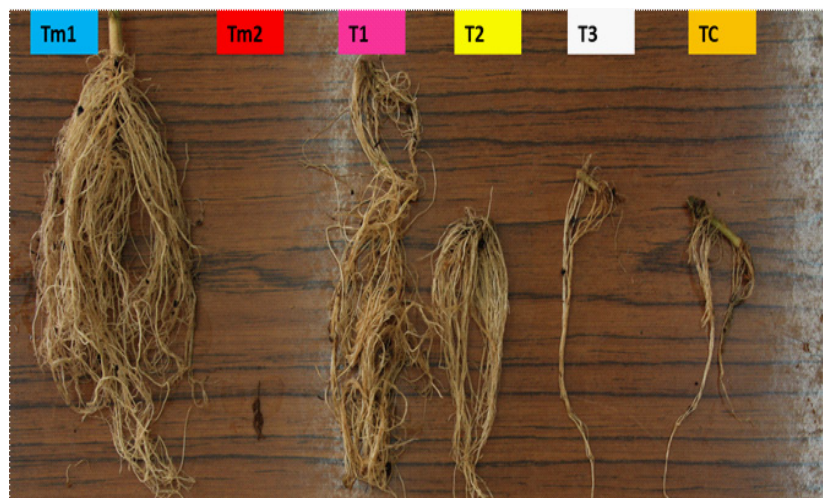

Figure 5. Roots of eggplant artificially infested with Verticllium and treated with different Trichoderma spores suspenssions; T. afro-harzianum in T1 treatment, T. reseei in T2, T. guizouhense in T3, and TC Trichoderma extracted from commercial product compared to controls; healthy eggplants Tm1 and eggplant inoculated with Verticillium only Tm2.

(2011) assessed 33 fungal antagonists including Trichoderma spp. with biocontrol potential in vitro which displayed efficacy under green house (Zheng et al., 2011). Carrero-Carron et al. (2016) have demonstrated that two of T. asperellum strains reduce significantly the severity of defoliating Verticilium disease on olive plants and promoting growth in infested and non-infested olive plants (Carrero-Carron et al., 2016). These interesting biocontrol properties and potential of Trichoderma spp. on root disease like Verticillium investigated in the previous and the present studies may be due to a number of reasons including Trichoderma antagonistic traits and inoculation method applied.

As mentioned in the literature investigating the naturally occurring highly susceptible eggplant and virulent Verticillium interactions allowed us to reveal the congruent potential 
of Trichoderma species like T. afro-harzianum in this work and exhibiting the antagonistic potential in reducing virulence of such pathogen. Another point, Trichoderma has been previously demonstrated to be used as compatibly as efficacious in biocontrol when combined with other BCAs.

Thaloromyces flavus and non pathogenic Fusarium oxysporum have been recognized as the most effective fungal biocontrol agents against Verticillium dahliae disease. In fact, different research work demonstrated the potential of different antagonists like Thalaromyces flavus, Fusarium oxysporum and phomopsis sp. to control different Verticillium wilt in tomatoes, pistachio, cotton and eggplant (Marois et al., 1982; Tjamos et al., 2004; Zheng et al., 2011; Angelopoulou et al., 2014). Recently Yuan et al (2017) research work on biocontrol efficacy of Penicillium simplicissimum with DI $=41.4 \%$, Acremonium $\mathrm{sp}$ with DI $=39.2 \%$, Leptosphaeria sp. with DI $=32.4 \%$, and Talaromyces flavus with DI $=36.9 \%$ against Verticillium disease showed effective control of the disease when seed soaked with these fungal antagonists (Yuan et al., 2017).

Therefore, combination of Trichoderma spp. with Thaloromyces flavus, non-pathogenic Fusarium oxysporum or Penicillium simplicissimum may be used as an integrated biocontrol application against Verticillium dahliae.

\section{Acknowledgments}

This research was supported by ARIMNET, PESTOLIVE project.

\section{References}

Angelopoulou DJ, EJ Naska, EJ Paplomatas, and SE Tjamos (2014) Biological control agents (BCAs) of Verticillium wilt: influence of application rates and delivery method on plant protection, triggering of host defense mechanisms and rhizosphere populations of BCAs. Plant Pathology 63 (5): 1062-1069.

Atibalentja N, and DM Eastburn (1997) Evaluation of Inoculation Methods for Screening Horseradish Cultivars for Resistance to Verticillium dahliae. American Phytopathology Society. 81 (4): 356-362.

Blestos F, C Thanassoulopoulous, and D Roupakias (2003) Effect of grafting on growth yield and Verticillium wilt of eggplant. Horticultural science 38 (2): 183-186.

Benson DM and KF Baker (1974) Epidemiology of Rhizoctonia solani preemergence damping-off of radish: inoculum potential and disease potential interaction. Phytopathology 64: 957-962.

Breda NJ (2003) Ground-based measurements of leaf area index: a review of methods, instruments and current controversies. Journal of Experimental Botany 54 (392): 2403-2417.

Carrero-Carron I, JL Trapero-Casas, CO García, E Monte, R Hermosa, and RM Jimenez-Díaz (2016) Trichoderma asperellum is effective for biocontrol of Verticillium wilt in olive caused by the defoliating pathotype of Verticillium dahliae. Crop protection 88: 45-52.

Cook RJ and KF Baker (1983) The nature and practice of biological control of plant pathogens. St Paul (MN). American Phytopatholgy Society, pp. 151-152.

Campbell CL and DA Neher (1994) Estimating disease severity and incidence. In: CL Campbell and DM Benson (Eds.) Epidemiology and Management of Root Diseases. Springer, New York, pp. 117-
147.

Dongfang H, W Chunsheng, T Fei, C Qian, X Xiangming, S Wenjing, and H Xiaoping (2014) Whole Genome Wide Expression Profiles on Germination of Verticillium dahliae Microsclerotia. Plos ONE 9 (6): e100046.

Gray LE, LA Achenbach, RJ Duff, and D Lightfoot (1999) Pathogenicity of Fusarium solani f. sp. glycines isolates on Soybean and Green Bean. Plants Journal of pathology 147 (5): 281-284.

Huisman OC and JS Gerik (1989) Dynamics of Colonization of Plant Roots by Verticillium Dahliae and Other Fungi. In: EC Tjamos and $\mathrm{CH}$ Beckman (eds) Vascular wilt disease of plant NATO ASI Series H. Cell biology, Springer-Verlag, pp 1-17.

Lumsden RD, JA Lewis, and PD Millner (1983) Effect of composted sewage sludge on several soilborne pathogens and diseases. Phytopathology 73: 1543-1548.

Marois JJ, SA Jahnson, MT Dunn, and GC Papavizas (1982) Biological control of Verticillium wilts of eggplant in the field. Plant Disease 6 (12): 1166-1168.

Mokhtari W, N Chtaina, E Halmschlager, H Volgmayr, C stauffer, and W Jaklitsch (2017) Potential antagonism of some Trichoderma strains isolated from Moroccan soil against three phytopathogenic fungi of great economic importance. Revue Marocaine des Sciences Agronomiques et Vétérinaires 5 (3): 248-254.

Monte E (2001) Understanding Trichoderma: between biotechnology and microbial ecology. International Journal of Microbiology 4: $1-4$.

Pegg GF and BL Brady (2002) Pathogenesis In: Pegg GF and BL Brady (eds) Verticillium wilts. Center for Agriculture and Biosciences International Publishing, Wallingford UK, pp 142-144.

Sang MK, OJ Yeon, and KD Kim (2007) Root dipping application of antagonistic rhizobacteria for the control of Phytophthora blight of pepper under field conditions. Plant pathology 23 (2): 109-112.

Schnathorst WC and GS Sibbett (1971) T-1 Verticillium strain: major factor in cotton and olive wilt. California Agriculture 25 (7): 3-5.

Shiraishi T, S Hiroshi, I Kentaro, and U Toshihiko (2014) A useful method for preparing microsclerotia inoculum of Verticillium dahliae. Journal of General Plant Pathology 80 (6): 475-478.

Tjamos EC (1981) Virulence of Verticillium dahliae and V. albo-atrum isolates in Tomato seedlings in relation to their host of origin and the applied cropping system. Phytopathology 71: 98-100.

Tjamos EC, DI Tsitsigiannis, SE Tjamos, PP Antoniou, and P Katinakis (2004) Selection and screening of endorhizosphere bacteria from solarized soils as biocontrol agents against Verticillium dahliae of solanaceous hosts. European Journal of Plant Pathology 110 (1): 35-44.

Van S (1980) Phytophthora root rot of sweet pepper. Netherland Journal of plant pathology 86 (5): 259-264.

Yang ZS, GH Li, PJ Zhao, X Zheng, SL Luo, L Li, XM Niu, and KQ Zhang (2010) Nematicidal activity of Trichoderma spp. and isolation of an active compound. World Journal of Microbiology and Biotechnology 26 (12): 2297-2302.

Yuan Y, F Hongjie, W Lingfei, L Zhifang, S Yongqiang, Z LiHong, F Zili, and Z Heqin (2017) Potential of Endophytic Fungi Isolated from Cotton Roots for Biological Control against Verticillium Wilt Disease. PLos ONE 12 (1): e0170557.

Yuliar Y, AN Yanetri, and T Koki (2015) Recent Trends in Control Methods for Bacterial Wilt Diseases Caused by Ralstonia solanacearum. Microbes Environment 30 (1): 1-11.

Zheng Y, QY Xue, LL Xu, Q Xu, S Lu, C Gu, and JH Guo (2011) A screening strategy of fungal biocontrol agents towards Verticillium wilt of cotton. Biological Control 56 (3): 209-216. 